PAPER

\title{
The time course of visual field recovery following transphenoidal surgery for pituitary adenomas: predictive factors for a good outcome
}

\author{
K K Gnanalingham, S Bhattacharjee, R Pennington, J Ng, N Mendoza
}

J Neurol Neurosurg Psychiatry 2005;76:415-419. doi: 10.1136/jnnp.2004.035576

See end of article for authors' affiliations

......................

Correspondence to: Mr Kanna K

Gnanalingham,

Brindhaven, II Deena

Close, Queens Drive

London W3 OHR, UK;

kannagnana@

doctors.org.uk

Received 7 January 2004

In revised form 3 July 2004

Accepted 7 July 2004

\begin{abstract}
Objective: To report the quantitative assessment of visual fields (VF) in patients with pituitary macroadenomas, and the time course and predictive factors for recovery of vision.

Methods: Retrospective study of 41 patients with pituitary adenomas and visual disturbance. Patients underwent pre- and postoperative VF assessment at one week, three to six months, one year, two years, and five years using the Humphrey field analyser, which gives a quantitative measure of VF in each quadrant.

Results: $36 / 41$ patients (88\%) presented with a visual disturbance. Mean (SEM) duration of symptoms was 94 (50) weeks (range 0.5 to 1500); 12 (29\%) had optic atrophy at presentation. Impairment of VF was greatest in the upper temporal quadrant, followed by the lower temporal. VF recovery was progressive and apparent even at the five year follow up $(p<0.001)$. Overall, VF returned to normal in $35 \%$ of eyes, improved in $60 \%$, and remained unchanged in 5\%. Patients whose VF returned to normal had a shorter duration of symptoms ( 16 (5) v 137 (56) weeks; $p<0.05$ ), better preoperative visual acuity $(p<0.05)$, and a smaller degree of impairment in preoperative lateral quadrant VF $(p<0.01)$ than those whose VF only improved. On multivariate analysis, the only predictive factor for VF recovery was the degree of impairment in VF preoperatively.

Conclusions: Transphenoidal surgery for pituitary macroadenoma results in a progressive recovery of VF in $95 \%$ of patients. The extent of the VF recovery is mainly dependent on the preoperative VF deficit, which emphasises the need for early intervention in these patients.
\end{abstract}

limina patients with pituitary tumours, the presence of visual field (VF) deficits is a major indication for surgical intervention, by the transcranial or transphenoidal route. ${ }^{1-3}$ Several studies have reported on the recovery in VF deficit following surgery for pituitary tumours. ${ }^{1-10}$ In general, the results have been positive, with improvement in vision in up to $90 \%$ of patients. ${ }^{34-9}$ However, the time course of the recovery of VF in these patients is not clear, although there is anecdotal evidence to suggest that recovery may extend for several years. ${ }^{4}{ }^{8}$ Moreover, the assessment of visual recovery has been qualitative (that is, categorised as improved, not improved, or returned to normal $)^{15}$ or semiquantitative, using a variety of scoring systems. ${ }^{4810}$ In an attempt to produce a more quantitative assessment of visual dysfunction, an American Medical Association committee devised a scoring system. ${ }^{11}$ This is a complex method that combines the corrected visual acuity (VA) and the degrees of peripheral vision for each eye along the eight radii, to provide a percentage of impairment of vision. ${ }^{311}$

We report a more quantitative assessment of VF in patients with pituitary macroadenomas, and the time course and predictive factors for postoperative recovery. In this study, VF was assessed using the Humphrey field analyser, which provides a fully quantitative assessment of VF with adjustments for changes caused by increasing age and the presence of cataracts.

\section{METHODS}

Patients admitted to our neurosurgical unit with pituitary neoplasms between 1995 and 1998 were retrospectively reviewed. Patients with preoperative impairment of VF, undergoing transphenoidal surgery for pituitary adenomas, and with at least five years of adequate follow up were included. Presenting symptoms and the duration of symptoms at presentation were recorded.

All patients underwent transphenoidal surgery of pituitary adenomas, using an endonasal approach as described previously. ${ }^{4}$ The extent of the surgical excision of tumour (total or partial) was recorded, as judged on postoperative magnetic resonance imaging. Patients with incomplete resection of tumour and those with tumour recurrence were referred for radiotherapy.

\section{Visual assessment}

Each patient underwent assessment by an ophthalmologist pre- and postoperatively (at two years), and findings such as optic atrophy (total disc pallor) and VA (using the Snellen chart) were recorded.

Patients also underwent preoperative and postoperative VF assessment at one week, three to six months, one year, two years, and five years, using the Humphrey field analyser II (30-2 test; Humphrey Instruments, London, UK). ${ }^{12}{ }^{13}$ In this automated test, the patient is required to fixate at a central point with each eye, while a light of variable intensity is flashed in the peripheral field of vision. The patient is required to acknowledge the flashing light by pressing a button. An experienced orthoptist gave the instructions and was present throughout the session, instructing the patient to refixate if fixation began to wonder. The Humphrey field analyser provides a quantitative measure of VF in each of the four quadrants, and the average reading for VF in each quadrant of the left and right eyes was recorded. The pattern deviation format of VF was used, which represents the VF in the patient adjusted for changes caused by increasing age, the

Abbreviations: VA, visual acuity; VF, visual field 
presence of cataracts, and small pupils. The Humphrey field analyser also records the reliability of each test, defined as less than $20 \%$ fixation losses and less than $33 \%$ false positive and false negative errors. ${ }^{12}{ }^{13}$ Only reliable tests were included in the study.

\section{Statistical analysis}

The postoperative changes in VF in each quadrant of the left and right eyes were analysed separately (that is, $n=41$ per eye), using repeated measures analysis of variance (ANOVA). At the five year follow up visit, the VF in each quadrant of each eye was compared with the preoperative VF and categorised as "improved," "returned to normal" (less than -5 dB VF deficit), "worse," or "not changed."

To evaluate the predictive factors for recovery in VF, univariate statistical analysis was carried out using the $\chi^{2}$ test (with Yates's correction) or the Mann-Whitney U test for categorical variables (that is, the presence of optic atrophy,

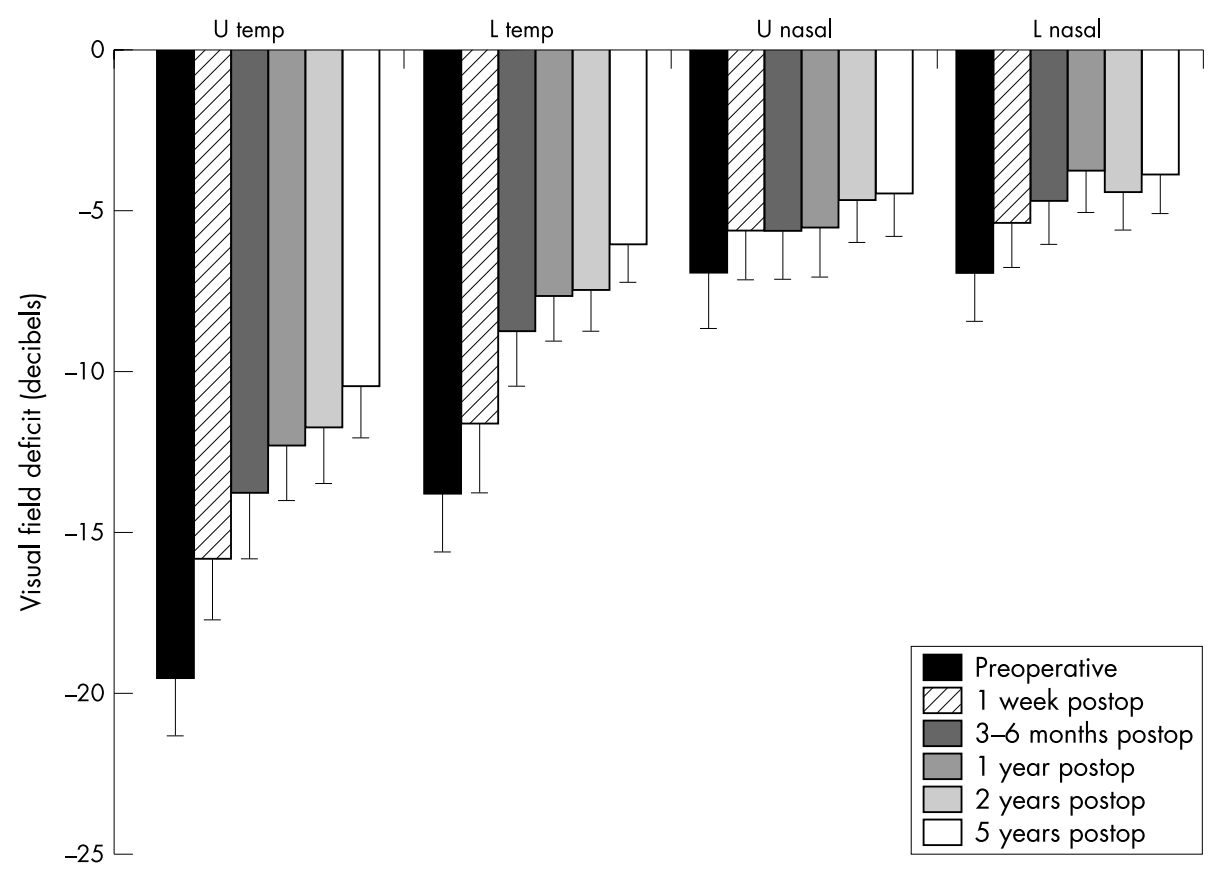

Figure 1 Postoperative changes in visual fields in each quadrant of the eyes following transphenoidal surgery $(n=82$ eyes; error bars $=S E M)$. L, lower; postop, postoperative; temp, temporal; U, upper.

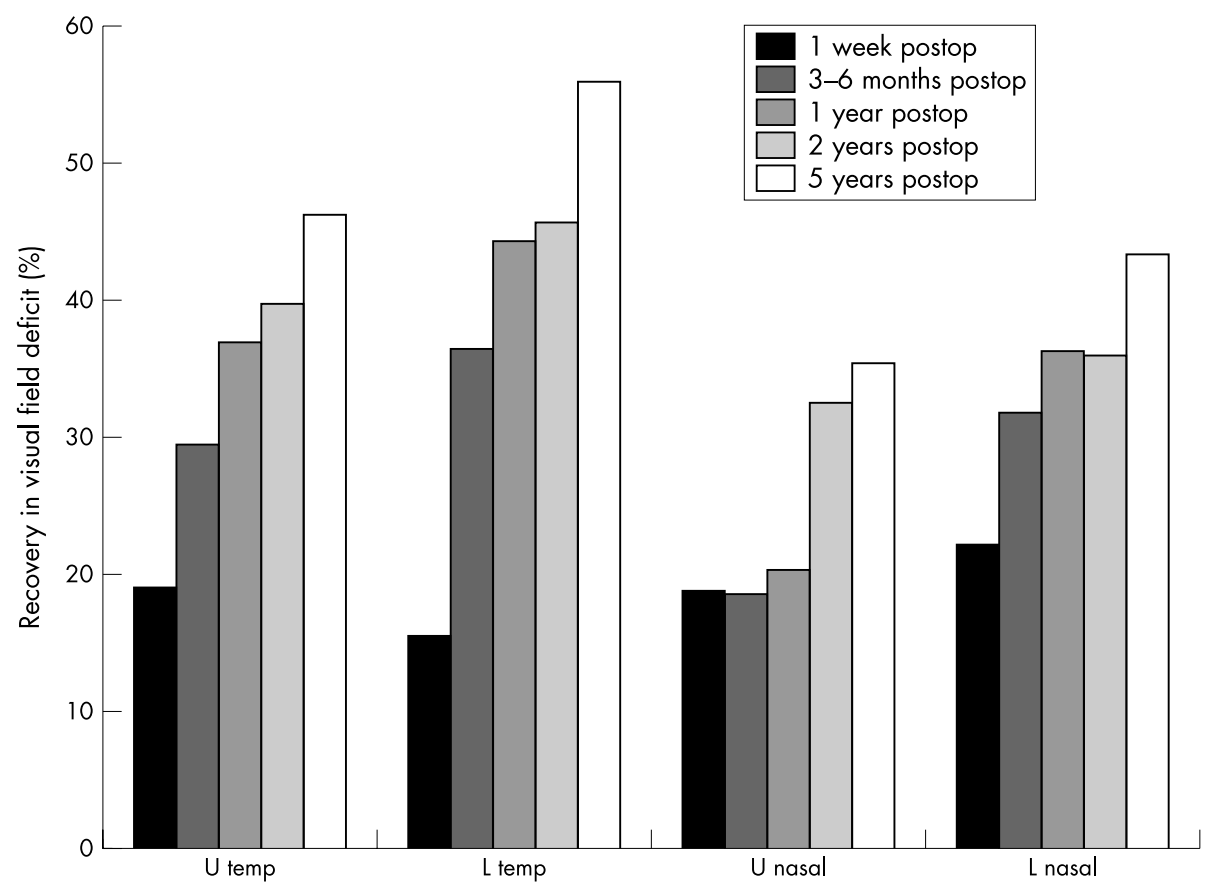

Figure 2 Percentage recovery in each quadrant of the visual fields of left and right eyes, at one week, three to six months, one year, two years, and five years following transphenoidal surgery $(n=82$ eyes; error bars $=S E M)$. The percentage recovery in VF at any time postoperatively was expressed as a percentage change in VF from the preoperative value. L, lower; postop, postoperative; $U$, upper. 
degree of tumour removal, visual acuity) and ANOVA for normal variables (patient's age, duration of visual symptoms). For multivariate analysis, a logistic regression model was used to determine the confounding effects of these factors on the recovery of VF at the five year follow up. In this model, recovery of VF (categorised as improved or returned to normal) was the dependent variable and the other confounding factors were entered as covariates in a forward stepwise manner. The correlation between the preoperative duration of symptoms and the degree of VF deficit was analysed using Pearson's test.

Post hoc pairwise comparisons were carried out with Bonferroni's test. The null hypothesis was rejected at a significance level of $\mathrm{p}<0.05$. All tests were done using the SPSS statistical package (Statistical Programs for the Social Sciences, Chicago, Illinois, USA).

\section{RESULTS}

\section{Demographic details}

Of the 56 patients available for study, 41 met the inclusion criteria, including follow up for five years or more. Their mean (SEM) age was 56 (2) years (range 30 to 80) and 21 $(51 \%)$ were male. The main presenting symptoms were visual disturbance $(\mathrm{n}=36)$ and endocrine disorder (prolactinoma or pituitary apoplexy, $n=5$ ). The mean (SEM) duration of symptoms preoperatively was 94 (50) weeks (range 0.5 to $1500)$. Optic atrophy was noted in 12 patients $(29 \%)$ at presentation.

The main operating surgeons were consultants $(\mathrm{n}=34)$ or senior trainees $(\mathrm{n}=7)$. Histopathologically, 37 patients (90\%) had non-functioning macroadenomas and 25 (61\%) had a macroscopic clearance of tumour. Postoperative radiotherapy was given to 14 patients (34\%) and three (7\%) underwent further surgery for tumour recurrence (one frontal craniotomy; two by the transphenoidal approach), at two, three, and three years, respectively, following the initial operation.

As there were major differences between the left and right eyes in terms of the preoperative deficits in VF and VA and the changes postoperatively, the results for the left and right eyes were treated individually (that is, 41 patients and 82 eyes).

\section{Changes in VA}

Preoperatively, VA was abnormal (6/9 or worse) in 57 eyes (70\%), and the median visual acuity was 6/9 (range 6/4 to $6 / 60$ ). Postoperatively, at the two year follow up, VA was abnormal in 30 eyes (37\%) and the median visual acuity had improved to $6 / 6$ (range $6 / 4$ to $6 / 60 ; \mathrm{p}<0.01$; Wilcoxon signed ranks test).

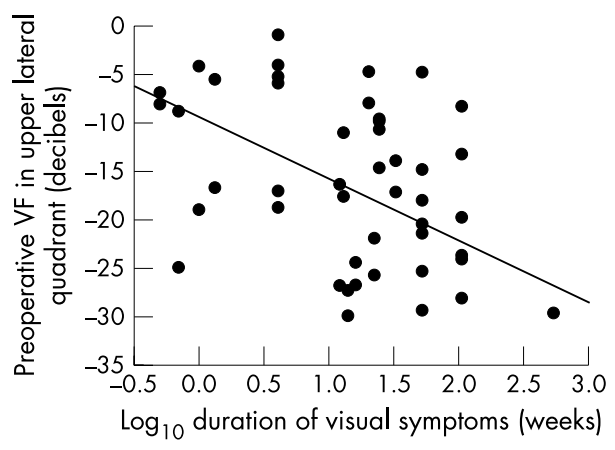

Figure 3 The logarithmic relation between the preoperative visual field (VF) deficit in the upper lateral quadrant and the duration of visual symptoms preoperatively ( $\log _{10}$ weeks) ( $n=82$ eyes; Pearson correlation, $r=-0.5 ; p<0.001)$.

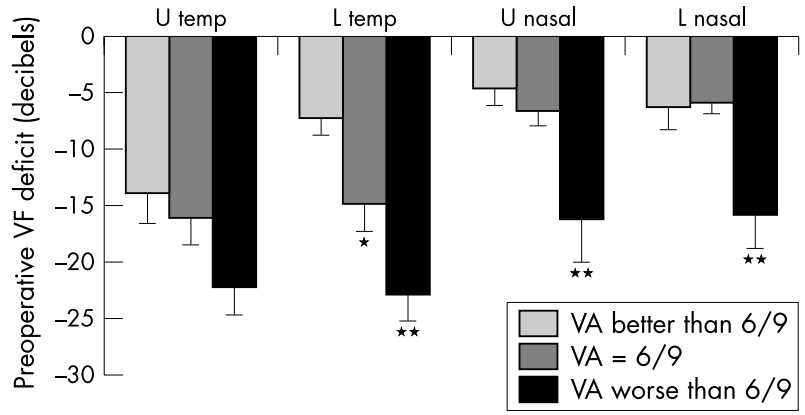

Figure 4 Relation between the preoperative visual field (VF) deficit and the preoperative visual acuity (VA) in each quadrant of the eye following transphenoidal surgery. Error bars $=$ SEM. The preoperative VA has been categorised about the median value of $6 / 9$ as better than $6 / 9$ $\left(n=37\right.$ eyes), 6/9 $(n=27)$, or worse than $6 / 9(n=18)$. ${ }^{*} p<0.05$; ${ }^{* *} p<0.01$ compared with VA better than the 6/9 group; one way ANOVA and post hoc Bonferroni's test. L, lower; U, upper.

\section{Changes in VF}

All 82 eyes had abnormal VF preoperatively, and impairment of the VF was greatest in the upper temporal quadrant, followed by the lower temporal and nasal quadrants (fig l). Recovery of VF was progressive in all four quadrants and apparent even at the five year follow up $(\mathrm{F}=13 ; \mathrm{p}<0.0001$; repeated measures ANOVA; fig 1). Overall, VF returned to normal in 29 eyes (35\%), improved in 49 (60\%), and remained unchanged in four $(5 \%)$. VF was not made worse in any of the eyes.

At the five year follow up, the percentage recovery of VF (expressed as percentage change in VF from the preoperative value) ranged from $35 \%$ to $56 \%$ (fig 2 ). In each quadrant of the VF, more than $50 \%$ of the eventual recovery had taken place by three to six months postoperatively (fig 2).

There was a logarithmic relation between the preoperative VF deficit in the upper lateral quadrant and the duration of visual symptoms preoperatively ( $\log _{10}$ weeks) $(\mathrm{n}=82$ eyes; Pearson correlation, $r=-0.5$; $\mathrm{p}<0.001$ ) (fig 3 ). The preoperative VF deficit was worse in patients with poor preoperative VA, categorised about the median value of $6 / 9$ $(\mathrm{p}<0.01$; one way ANOVA and post hoc Bonferroni test; fig 4). There was no relation between the duration of symptoms preoperatively and the preoperative VA $(\mathrm{F}=1.7$; $\mathrm{p}=0.2$; one way ANOVA).

\section{Predictive factors for recovery in VF}

On univariate analysis, the patients whose VF returned to normal had a shorter duration of symptoms (16 (5) $v 137$ (56) weeks; $\mathrm{F}=5$; $\mathrm{p}<0.05)$ and better VA $(\mathrm{p}<0.05$; MannWhitney $U$ test) than patients whose VF only improved (table 1). There was no difference in age, presence of optic atrophy, degree of tumour excision, or postoperative radiotherapy between these groups (table 1). The patients whose VF returned to normal had a smaller degree of impairment in the preoperative VF in the upper and lower temporal quadrants than patients whose VF only improved $(\mathrm{p}<0.01$; repeated measures ANOVA; fig 5).

On multivariate analysis with logistic regression, the only predictor for recovery of VF at the five year follow up (categorised as returned to normal or improved) was severity of preoperative VF deficit in the upper temporal quadrant (odds ratio $=1.2 ; 95 \%$ confidence interval, 1.04 to 1.4 ; $\mathrm{p}=0.01$ ). None of the other factors (age, duration of symptoms preoperatively, operating surgeon, presence of optic atrophy, preoperative visual acuity, extent of surgical resection of tumour, or postoperative radiotherapy) had a significant influence on recovery of VF $(\mathrm{p}>0.5)$. 
Table 1 Factors affecting the postoperative recovery in visual field deficits

\begin{tabular}{lll}
\hline Variable & $\begin{array}{l}\text { VF returned } \\
\text { to normal }\end{array}$ & VF improved \\
\hline $\mathrm{n}(\%)$ & $29(36 \%)$ & $49(60 \%)$ \\
Age (years) & $54.1(3.0)$ & $55.8(2.0)$ \\
$\begin{array}{l}\text { Duration of symptoms preoperatively } \\
\text { (weeks) }\end{array}$ & $17(5)$ & $137(56)^{*}$ \\
$\begin{array}{l}\text { Preoperative visual acuity (median) } \\
\text { Presence of optic atrophy (\%) }\end{array}$ & $6 / 6$ & $6 / 9^{*}$ \\
$\begin{array}{l}\text { Macroscopic clearance of tumour (\%) } \\
\text { Postoperative radiotherapy (\%) }\end{array}$ & $16(24 \%)$ & $15(31 \%)$ \\
\hline
\end{tabular}

Values are mean (SEM) or $\mathrm{n}(\%)$. The recovery in VF deficits was classified as improved ( $n=49$ eyes) or returned to normal ( $n=29$ eyes).

${ }^{*} \mathrm{p}<0.05 v \mathrm{VF}$ returned to normal; unpaired Student's $t$ test or Mann-

Whitney $U$ test.

$\mathrm{VF}$, visual fields.

\section{DISCUSSION}

Impairment of visual fields is an important indication for surgery in pituitary lesions. ${ }^{1-4}$ In patients with pituitary adenomas, the time course of recovery in VF after surgery is unclear. We studied the recovery in VF in such patients quantitatively and derived predictive factors for a good outcome. In the present study, VF was assessed using the Humphrey field analyser, which not only provides a fully quantitative assessment of VF but also incorporates tests for patient reliability and adjustments for changes in VF caused by increasing age, the presence of cataracts, and small pupils. $^{12}{ }^{13}$ In addition, the Humphrey field analyser is automated and thus less prone to examiner bias than other non-automated methods of assessing VF..$^{12}$

\section{Time course of recovery of VF}

The main findings of our study were that the preoperative deficit in VF was greater in the temporal quadrants and that postoperatively there was progressive recovery of VF in all quadrants, which extended to five years or more. Overall, VF returned to normal or improved in $95 \%$ of eyes.

Our study also demonstrates in a quantitative fashion that the recovery of VF is progressive over several years and that most of it (more than 50\%) takes place in the first three to six months postoperatively. This is an important finding from a prognostic point of view. Previous studies have either failed to consider the postoperative period as a factor, or studied the recovery of VF over a much shorter follow up period. ${ }^{13-8}$ Nevertheless, our observations are consistent with the anecdotal evidence that recovery of VF may be evident from the immediate postoperative period and may extend for several years. ${ }^{1} 47810$

Our results compare favourably with other larger surgical series employing the transphenoidal approach, which have reported postoperative improvement or normalisation of VF in $74-90 \%$ of patients. ${ }^{34-8}$ Some series have also noted deterioration in vision in a minority of patients (4-5\%) when using the transphenoidal technique. ${ }^{347}$ Comparison of outcome between these studies with respect to recovery of VF is limited by the differences in the methodology employed. Some scoring systems for assessment of VF have been entirely qualitative, ${ }^{156}$ while others have been semiquantitative,,$^{4810}$ or complex in nature. ${ }^{311}$ There is a need for a standard method for assessment of VF that is simple, reliable, and automated, such as the Humphrey fields.

\section{Predictive factors for recovery of VF}

On univariate analysis, better recovery of VF was associated with a shorter duration of symptoms and a smaller

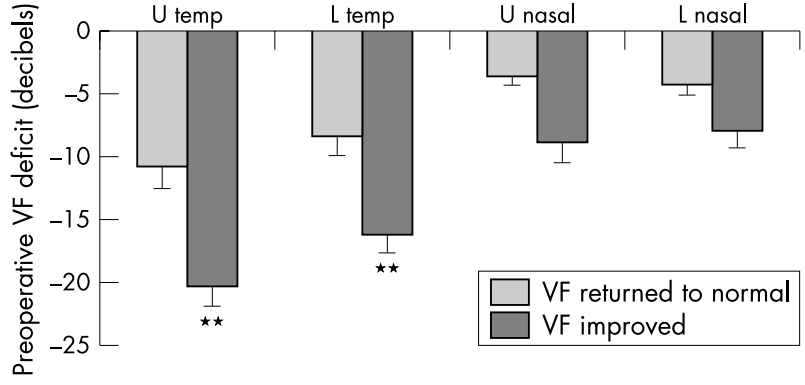

Figure 5 The relation between the preoperative visual field (VF) deficit and the postoperative recovery of VF in each quadrant of the eye following transphenoidal surgery. Error bars $=$ SEM. The postoperative recovery of VF was classified as improved ( $n=49$ eyes) or returned to normal ( $n=29$ eyes). ${ }^{* *} p<0.01$; repeated measures ANOVA and post hoc Bonferroni's test. L, lower; U, upper.

preoperative deficit in VA and VF. However, the preoperative deficit in VF was also related to both the duration of symptoms and the preoperative VA. In this retrospective study, we employed a logistic regression model to evaluate the influence of these confounding factors on VF recovery. On multivariate analysis, the only independent predictor of postoperative recovery of VF was the degree of preoperative VF deficit. This emphasises the need for prompt diagnosis of visual deterioration and early intervention in patients with pituitary adenomas.

There have been contradictory findings in previous studies regarding predictive factors for recovery of vision in these patients. Thus a shorter duration of symptoms, younger age, and a smaller deficit in preoperative VA have been reported to be associated with better postoperative recovery of VF by some investigators ${ }^{10}$ but not others. ${ }^{48}$ Likewise, the association between a smaller deficit in preoperative VF and a greater postoperative recovery of VF is not a universal finding. ${ }^{7810}$ The reason for this inconsistency is not clear, but it may be related to differences in the patient population studied, including differences in preoperative deficits in VF and VA, the extent of tumour resection, and the dose of postoperative radiotherapy. ${ }^{410}$ Furthermore, as apparent in the present study, preoperative deficits in VF and VA are interrelated, and multivariate statistical analysis to adjust for multiple confounding factors was not carried out in previous reports. Consistent with our findings, optic atrophy was not found to be a predictive factor for VF recovery. ${ }^{4}{ }^{10}$ Although not investigated in the present study, others have noted the relative lack of importance of the size of the pituitary adenoma in determining visual recovery. ${ }^{16}$

\section{Conclusions}

This study confirms that the recovery of VF deficit following transphenoidal surgery in patients with pituitary macroadenomas takes place over many years. The greatest influence on VF recovery was the extent of deficit in VF preoperatively, which has important clinical implications.

\section{ACKNOWLEDGEMENTS}

We gratefully acknowledge the assistance of the orthoptists and Dr Valerie Ferguson, consultant ophthalmologist, at Charing Cross Hospital.

\section{Authors' affiliations}

K K Gnanalingham, S Bhattacharjee, R Pennington, J Ng, N Mendoza, Department of Neurosurgery, Charing Cross Hospital, Fulham Palace Road, London W4, UK

Competing interests: none declared 


\section{REFERENCES}

1 Symon L, Jakubowski J. Transcranial management of pituitary tumour with suprasellar extension. J Neurol Neurosurg Psychiatry 1979;42:973-82.

2 Fahlbusch R, Honegger J, Buchfelder M. Surgical management of acromegaly. Endocr Metab Clin North Am 1992;21:669-92.

3 Laws ER, Trautmann JC, Hollenhorst RW. Trans-sphenoidal decompression of the optic nerve and chiasm. J Neurosurg 1977;46:717-22.

4 Powell M. Recovery of vision following trans-sphenoidal surgery for pituitary adenomas. Br J Neurosurg 1995;9:367-73.

5 Svien HJ, Love JG, Kennedy WC, et al. Status of vision following surgical treatment for pituitary chromophobe adenoma. J Neurosurg 1965:22:47-52.

6 Ciric I, Mikhael M, Stafford T, et al. Trans-sphenoidal microsurgery of pituitary macroadenomas with long-term follow-up results. J Neurosurg 1983;59:395-401.

7 Findlay G, McFadzean RM, Teasdale G. Recovery of vision following treatment of pituitary tumours: application of a new system of assessment to patients treated by trans-sphenoidal operation. Acta Neurochir 1983;68:175-86.

8 Peter M, Tribolet ND. Visual outcome after transphenoidal surgery for pituitary adenomas. Br J Neurosurg 1995;9:151-7.

9 Trautmann JC, Laws ER. Visual status after transphenoidal surgery at the Mayo Clinic 1971-1982. Am J Ophthalmol 1983;96:200-8.

10 Cohen AR, Cooper PR, Kupersmith MJ, et al. Visual recovery after trans-sphenoidal removal of pituitary adenomas. Neurosurgery 1985; 17:446-52

11 Committee on Medical Rating of Physical Impairment. Guides to the evaluation of permanent impairment. The visual system. JAMA 1958; 168:475-88.

12 Enger C, Sommer A. Recognising glaucomatous field loss with the Humphrey STATPAC. Arch Ophthalmol 1987; 105:1355-7

13 Katz J, Sommer A. Reliability indexes of automated perimetric tests. Arch Ophthalmol 1988; 106:1252-4.

Committee on Publication Ethics Seminar 2005

Friday 11 March 2005, 9.30 am - 5 pm, BMA House, London

This year's seminar will focus on COPE's new Code of Conduct for Editors and interactive workshops on common ethical and editorial dilemmas. The seminar is for editors, authors, and all those interested in increasing the standard of publication ethics.

The Code aims to set a new basic standard for the ethical conduct of editors and sets out guidelines for quality and correcting the record, standing by decisions made, ethics committee approval, consent for publication confidentiality of submitted material, guidance to authors, pursuing misconduct, relationship to publishers, owners, and advertisers, and conflict of interest. The code also creates a mechanism to refer a complaint to COPE if an editor has breached the code.

The seminar will include:

- The new Code of Conduct for Editors

- Dr lona Heath, Chair BMJ Ethics Committee-research, audit, and ethics committee approval

- COPE's new website-full text and keyword searching for COPE's advice on specific issues, for example research misconduct, conflict of interest, and deception

- Interactive workshops-common ethical and editorial dilemmas for editors

- Opportunities to network with other editors and share your experiences and challenges

The seminar is free for COPE members and $£ 30.00$ for non-members. Numbers are limited and early booking is advisable. For registrations or more information please contact Sam Knottenbelt at cope@bmigroup.com or call 0207383 6602. For more information on COPE see www.publicationethics.org.uk/ 